PROCEEDINGS OF THE

AMERICAN MATHEMATICAL SOCIETY

Volume 128, Number 5, Pages 1257-1262

S 0002-9939(00)05678-1

Article electronically published on February 7, 2000

\title{
THE BIERI-NEUMANN-STREBEL INVARIANT FOR BASIS-CONJUGATING AUTOMORPHISMS OF FREE GROUPS
}

\author{
LISA A. ORLANDI-KORNER
}

(Communicated by Ronald M. Solomon)

\begin{abstract}
The pure symmetric automorphism group of the free group on $n$ generators, $P \Sigma_{n}$, consists of those automorphisms which take each generator to a conjugate of itself. We describe the Bieri-Neumann-Strebel invariant of $P \Sigma_{n}$, which determines, among other things, which subgroups containing the commutator are finitely generated.
\end{abstract}

\section{INTRODUCTION}

Let $F_{n}$ be the group freely generated by the set $X_{n}=\left\{x_{1}, \ldots, x_{n}\right\}$. The pure symmetric automorphisms of $F_{n}$ are those automorphisms $\alpha$ such that $\alpha\left(x_{i}\right)$ is conjugate to $x_{i}$, for $i=1, \ldots, n\left[3\right.$. They form the group $P \Sigma_{n}$, a subgroup of $\operatorname{Aut}\left(F_{n}\right)$ which can also be described as the group of motions of $n$ unknotted, unlinked circles in $\mathbf{R}^{3}$ [5]. McCool found a finite presentation for $P \Sigma_{n}$ [8], whose generators are the automorphisms $\alpha_{i j}$ (for $i \neq j$ ), where $\alpha_{i j}\left(x_{i}\right)=x_{j}^{-1} x_{i} x_{j}$ and $\alpha_{i j}\left(x_{k}\right)=x_{k}$ if $k \neq i$. The defining relations come in three types:

1. $\left[\alpha_{i j}, \alpha_{k l}\right]=1$ where $i, j, k, l$ are distinct,

2. $\left[\alpha_{i j}, \alpha_{k j}\right]=1$ where $i, j, k$ are distinct,

3. $\left[\alpha_{i j} \alpha_{k j}, \alpha_{i k}\right]=1$ where $i, j, k$ are distinct.

Normal forms for these basis-conjugating automorphism groups were recently presented by Gutiérrez and Krstić [6].

In this paper, we use group actions on $\mathbf{R}$-trees to determine the Bieri-NeumannStrebel invariant of $P \Sigma_{n}$. The BNS-invariant of a finitely generated group $G$ tells, among other things, which subgroups of $G$ with abelian quotient are finitely generated. Its introduction in a 1987 issue of Inventiones [1] was accompanied by an alternative characterization by Brown [2] using group actions on $\mathbf{R}$-trees. Meinert used this $\mathbf{R}$-tree approach to compute the Bieri-Neumann-Strebel invariants of graph products of groups [9].

\section{Group actions on R-TreEs AND THE BIERI-NEUMANN-STREBEL INVARIANT}

For detailed background information about group actions on $\mathbf{R}$-trees, consult [4]. See [7] for additional material relating the actions to BNS-invariants. Suppose that $G$ is a finitely generated group which acts by isometries on an $\mathbf{R}$-tree, $T$, with

Received by the editors March 15, 1998.

2000 Mathematics Subject Classification. Primary 20F28; Secondary 20 E08.

(C)2000 American Mathematical Society 
metric $d$. Then the length function $l: G \rightarrow \mathbf{R}^{+}$is defined by $l(g)=\inf _{x \in T} d(x, g x)$. For every element $g$, this length is achieved on the characteristic subtree of $g$, $C_{g}=\{x \in T \mid d(x, g x)=l(g)\}$. If $l(g)=0$, then $g$ is elliptic and $C_{g}$ is its fixed set. If $l(g)>0$, then $g$ is hyperbolic and $C_{g}$ is its axis, isometric to $\mathbf{R}$, upon which it acts by translation. Two basic observations about characteristic subtrees will be extremely useful:

Fact $A$. If $g, h$ commute, and $h$ is hyperbolic, then $C_{g} \supseteq C_{h}$.

Fact B. If $g, h$ commute, then $C_{g h} \supseteq C_{g} \cap C_{h}$.

Assume further that the action of $G$ on $T$ is non-trivial and abelian; that is, $l$ is zero on $[G, G]$, but not on the whole group $G$. A non-trivial abelian action must fix an end $e$, so there exists a ray $R$ such that $C_{g} \cap R$ is unbounded for all $g \in G$. We can associate a homomorphism $\chi: G \rightarrow \mathbf{R}$ to the action, defined as follows:

$$
\chi(g)= \begin{cases}l(g) & \text { if } C_{g} \cap R \subseteq g\left(C_{g} \cap R\right), \\ -l(g) & \text { if } g\left(C_{g} \cap R\right) \subseteq C_{g} \cap R .\end{cases}
$$

In other words, $\chi(g)$ is positive if $g$ translates its axis away from $e$, negative if it translates toward $e$. If the action fixes two ends, then it has an invariant line which every element either translates or fixes pointwise. Such an action has two associated homomorphisms: $\chi$ and $-\chi$. If the action fixes only one end, then it has no invariant line and only one associated homomorphism; such an action is called exceptional.

If two $G$-actions on $\mathbf{R}$-trees differ only by metric dilation, then their associated homomorphisms are positive scalar multiples of one another. We will consider any two such homomorphisms equivalent. The resulting equivalence classes comprise the character sphere $S(G)$; thus when we discuss a character $\chi: G \rightarrow \mathbf{R}$, we will be referring to an equivalence class in $\operatorname{Hom}(G, \mathbf{R}) \backslash\{0\}$. We can now use Brown's characterization to describe the Bieri-Neumann-Strebel invariant $\Sigma(G)$ (often denoted by $\left.\Sigma^{1}(G)\right)$ :

Definition. A character $\chi$ in $S(G)$ is in the Bieri-Neumann-Strebel invariant $\Sigma(G)$ if and only if there is no exceptional abelian action associated to $\chi$.

It is worth noting that the action of $\operatorname{Aut}(G)$ on $S(G)$ given by $\chi \mapsto \chi \circ \phi$ leaves $\Sigma(G)$ invariant. Thus, we can use a subgroup of $\operatorname{Aut}\left(P \Sigma_{n}\right)$ to simplify our analysis of $\Sigma\left(P \Sigma_{n}\right)$. Every permutation $\sigma$ of $\{1, \ldots, n\}$ induces an automorphism of $P \Sigma_{n}$ defined by $\alpha_{i j} \mapsto \alpha_{\sigma(i) \sigma(j)}$. Denote by $P$ the subgroup of $A u t\left(P \Sigma_{n}\right)$ consisting of these permutation-induced automorphisms. Then the action of $P$ on $S\left(P \Sigma_{n}\right)$ will leave $\Sigma\left(P \Sigma_{n}\right)$ invariant, allowing us to permute the generators' indices on occasion.

Theorem. The complement of $\Sigma\left(P \Sigma_{n}\right)$ in $S\left(P \Sigma_{n}\right)$ consists of the following:

(1) for each pair of integers $\{i, j\}$ such that $1 \leq i<j \leq n$, those characters which map to 0 all generators except possibly $\alpha_{i j}$ and $\alpha_{j i}$, and

(2) for each triple of integers $\{i, j, k\}$ such that $1 \leq i<j<k \leq n$, those characters $\chi$ such that

$$
\begin{aligned}
& \chi\left(\alpha_{j i}\right)=-\chi\left(\alpha_{k i}\right), \\
& \chi\left(\alpha_{i j}\right)=-\chi\left(\alpha_{k j}\right), \\
& \chi\left(\alpha_{i k}\right)=-\chi\left(\alpha_{j k}\right),
\end{aligned}
$$

and $\chi$ maps all other generators to 0 . 
For example, $S\left(P \Sigma_{4}\right)$ is an 11-sphere; $\Sigma\left(P \Sigma_{4}\right)$ is $S\left(P \Sigma_{4}\right)$ minus six dimension-1 subspheres coming from (1), and four dimension-2 subspheres coming from (2).

Corollary. $\Sigma\left(P \Sigma_{n}\right)$ is dense in $S\left(P \Sigma_{n}\right)$ for $n \geq 3$.

\section{Proof of the Theorem}

It will be convenient for us to think of the generators $\left\{\alpha_{i j}\right\}$ of $P \Sigma_{n}$ as entries in an $n \times n$ matrix, $M$. The diagonal entries are thus undefined (since $\alpha_{i i}$ is undefined for $i=1, \ldots, n$ ), but we use the matrix only for organizational purposes. If $P \Sigma_{n}$ acts on an $\mathbf{R}$-tree, then the hyperbolic generators in any column of $M$ must share an axis, by Fact A. Lemmas 1 and 2 show that if $P \Sigma_{n}$ has an exceptional abelian action on an $\mathbf{R}$-tree, and if two hyperbolic generators share a column of $M$, then the two generators translate their common axis by the same distance, in opposite directions.

Lemma 1. Let $\chi$ be a character associated to an abelian action of $P \Sigma_{n}$ on an $\mathbf{R}$-tree. Suppose $\chi\left(\alpha_{21}\right) \neq 0$ and $\chi\left(\alpha_{31}\right) \neq 0$, and let $A$ be the common axis of $\alpha_{21}$ and $\alpha_{31}$. Then $C_{\alpha_{i j}} \supseteq A$ if $i \geq 4$ or $j \geq 4$.

Proof. We use Facts A and B to show the containment.

If $i \geq 4$, then $\alpha_{i j}$ commutes with $\alpha_{21}$ or $\alpha_{31}$, so $C_{\alpha_{i j}} \supseteq A$.

If $j \geq 4$ and $i \neq 1$, then $\alpha_{i j}$ commutes with $\alpha_{21}$ or $\alpha_{31}$, so $C_{\alpha_{i j}} \supseteq A$.

If $j \geq 4$ and $i=1$, then consider the product $\alpha_{2 j} \alpha_{i j}=\alpha_{2 j} \alpha_{1 j}$. Since it commutes with $\alpha_{21}, C_{\alpha_{2 j} \alpha_{i j}} \supseteq A$. We know by the case above that $C_{\alpha_{2 j}} \supseteq A$, so $C_{\alpha_{i j}} \supseteq$ $C_{\alpha_{2 j}} \cap C_{\alpha_{2 j} \alpha_{i j}} \supseteq A$.

Lemma 2. Let $\chi \in \Sigma\left(P \Sigma_{n}\right)^{c}$. Suppose $i, j, k$ are distinct such that $\chi\left(\alpha_{i j}\right) \neq 0$ and $\chi\left(\alpha_{k j}\right) \neq 0$. Then $\chi\left(\alpha_{i j}\right)=-\chi\left(\alpha_{k j}\right)$.

Proof. We assume that $\chi\left(\alpha_{i j}\right) \neq-\chi\left(\alpha_{k j}\right)$, and show that $\chi \in \Sigma\left(P \Sigma_{n}\right)$, contradicting our hypothesis. By permutation of indices, we may assume without loss of generality that $i=2, j=1, k=3$.

Consider an abelian action associated to $\chi$; we must show that it is not exceptional. We know that $\alpha_{21}$ and $\alpha_{31}$ are hyperbolic, and since $\chi\left(\alpha_{21}\right) \neq-\chi\left(\alpha_{31}\right)$, $\alpha_{21} \alpha_{31}$ must be hyperbolic as well. Therefore the three commuting elements must share an axis; call it $A$.

By Lemma $1 C_{\alpha_{i j}} \supseteq A$ if $i \geq 4$ or $j \geq 4$. Further, we can use Facts A and B to show that for all $i, j \in\{1,2,3\}, C_{\alpha_{i j}} \supseteq A$. Since $\alpha_{23}$ and $\alpha_{32}$ commute with $\alpha_{21} \alpha_{31}, C_{\alpha_{23}} \supseteq A$ and $C_{\alpha_{32}} \supseteq A$. Since $\alpha_{23} \alpha_{13}$ and $\alpha_{32} \alpha_{12}$ commute with $\alpha_{21}$ and $\alpha_{31}$, respectively, $C_{\alpha_{23} \alpha_{13}} \supseteq A$ and $C_{\alpha_{32} \alpha_{12}} \supseteq A$. Thus, $C_{\alpha_{13}} \supseteq C_{\alpha_{23}} \cap C_{\alpha_{23} \alpha_{13}} \supseteq A$ and $C_{\alpha_{12}} \supseteq C_{\alpha_{32}} \cap C_{\alpha_{32} \alpha_{12}} \supseteq A$.

Now we have shown that $A$ is invariant under the group action, so the action is not exceptional. Hence, $\chi \in \Sigma\left(P \Sigma_{n}\right)$.

Corollary. If $\chi \in \Sigma\left(P \Sigma_{n}\right)^{c}$, then each column of $M$ contains at most two generators with non-zero $\chi$-values.

Through the next lemma, we limit even further the number of generators with non-zero $\chi$-values, if $\chi \in \Sigma\left(P \Sigma_{n}\right)^{c}$.

Lemma 3. Let $\chi \in \Sigma\left(P \Sigma_{n}\right)^{c}$. If $i, j, k$, and $l$ are distinct, then $\chi\left(\alpha_{i j}\right)=0$ or $\chi\left(\alpha_{k l}\right)=0$. 
Proof. Assume the contrary, letting $i, j, k, l$ be distinct such that $\chi\left(\alpha_{i j}\right) \neq 0$ and $\chi\left(\alpha_{k l}\right) \neq 0$. Again, we consider an abelian action associated to $\chi$, and show that it is not exceptional, thereby contradicting our hypothesis that $\chi \notin \Sigma\left(P \Sigma_{n}\right)$. Since $\alpha_{i j}$ and $\alpha_{k l}$ are hyperbolic and commute with each other, they share an axis; call it $A$.

Every generator which commutes with $\alpha_{i j}$ or $\alpha_{k l}$ has a characteristic subtree which contains $A$. The only generators remaining have one index in common with each, but share the second index with neither: $\alpha_{i k}, \alpha_{j k}, \alpha_{k i}, \alpha_{l i}$.

Since $\alpha_{i k} \alpha_{j k}$ commutes with $\alpha_{i j}$, and $\alpha_{k i} \alpha_{l i}$ commutes with $\alpha_{k l}$, we know that $C_{\alpha_{i k} \alpha_{j k}} \supseteq A$ and $C_{\alpha_{k i} \alpha_{l i}} \supseteq A$. Hence, we get two equivalences from Fact B:

$$
\begin{aligned}
& C_{\alpha_{i k}} \supseteq A \Longleftrightarrow C_{\alpha_{j k}} \supseteq A, \\
& C_{\alpha_{k i}} \supseteq A \Longleftrightarrow C_{\alpha_{l i}} \supseteq A .
\end{aligned}
$$

We now consider the products $\alpha_{i j} \alpha_{k j}$ and $\alpha_{i l} \alpha_{k l}$.

If either is hyperbolic, then it has axis $A$ because it commutes with $\alpha_{i j}$ or $\alpha_{k l}$. In this case, $C_{\alpha_{i k}} \supseteq A$ and $C_{\alpha_{k i}} \supseteq A$, by Fact $\mathrm{A}$.

If both are elliptic, then $\chi\left(\alpha_{k j}\right)=-\chi\left(\alpha_{i j}\right) \neq 0$ and $\chi\left(\alpha_{i l}\right)=-\chi\left(\alpha_{k l}\right) \neq 0$, so by the Corollary to Lemma 2, $\chi\left(\alpha_{l j}\right)=0$ and $\chi\left(\alpha_{j l}\right)=0$. In this case, $\chi\left(\alpha_{i j} \alpha_{l j}\right) \neq 0$ and $\chi\left(\alpha_{k l} \alpha_{j l}\right) \neq 0$, so both $\alpha_{i j} \alpha_{l j}$ and $\alpha_{k l} \alpha_{j l}$ are hyperbolic with axis $A$. Hence, by Fact $\mathrm{A}, C_{\alpha_{l i}} \supseteq A$ and $C_{\alpha_{j k}} \supseteq A$.

In either case, the characteristic subtrees of $\alpha_{i k}, \alpha_{j k}, \alpha_{k i}$, and $\alpha_{l i}$ contain $A$. Therefore $A$ is invariant under the group action, so the action is not exceptional.

Now we can prove that the characters in $\Sigma\left(P \Sigma_{n}\right)^{c}$ are exactly those of types (1) and (2), described in the statement of the Theorem.

Proof $(\subseteq)$. Let $\chi: P \Sigma_{n} \rightarrow \mathbf{R}$ be a character in $\Sigma\left(P \Sigma_{n}\right)^{c}$. We will show that $\chi$ must be of type (1) or (2). The subsets of each type are invariant under the action of $P$ on $S\left(P \Sigma_{n}\right)$, so we may permute the indices of generators. Since $\chi \notin \Sigma\left(P \Sigma_{n}\right)$, we consider an exceptional abelian action of $P \Sigma_{n}$ on an $\mathbf{R}$-tree, associated to $\chi$.

Case 1. Each column of $M$ contains at most one hyperbolic generator.

Permute indices, if necessary, so that $\chi\left(\alpha_{21}\right) \neq 0$. Let $A$ be the axis of $\alpha_{21}$. We begin by showing that $A$ is contained in the characteristic subtrees of all the generators except $\alpha_{12}$.

For all $i, j \geq 3, \alpha_{i j}$ commutes with $\alpha_{21}$, so $C_{\alpha_{i j}} \supseteq A$. For the same reason, $C_{\alpha_{i 1}} \supseteq A$ and $C_{\alpha_{21} \alpha_{i 1}} \supseteq A$ for all $i \geq 3$. Further, since $\chi\left(\alpha_{i 1}\right)=0, \alpha_{21} \alpha_{i 1}$ is hyperbolic. This product commutes with $\alpha_{2 i}$ and $\alpha_{i 2}$, so $C_{\alpha_{2 i}} \supseteq A$ and $C_{\alpha_{i 2}} \supseteq A$, for all $i \geq 3$.

The only generators remaining are those of the form $\alpha_{1 i}$. If $i \geq 3$, then $\alpha_{2 i} \alpha_{1 i}$ commutes with $\alpha_{21}$, so $C_{\alpha_{2 i} \alpha_{1 i}} \supseteq A$. We have shown that $C_{\alpha_{2 i}} \supseteq A$, so it follows from Fact $\mathrm{B}$ that $C_{\alpha_{1 i}} \supseteq A$, as well. Our action is exceptional; therefore our only remaining generator, $\alpha_{12}$, must not leave $A$ invariant. Thus $C_{\alpha_{12}} \nsupseteq A$.

We are now in a position to show that $\chi$ kills all generators, except $\alpha_{21}$ and possibly $\alpha_{12}$. We know that $\chi\left(\alpha_{i 1}\right)=0$, for all $i \geq 3$. By Fact $\mathrm{A}$, we also know that $\chi\left(\alpha_{i 2}\right)=0$ for all $i \geq 3$; otherwise $\alpha_{i 2}$ would be hyperbolic with axis $A$, so $C_{\alpha_{12}} \supseteq A$, which is impossible. Further, by Lemma 3 $\chi\left(\alpha_{i j}\right)=0$ for all $i, j \geq 3$. The only remaining generators are of the forms $\alpha_{1 j}(j \geq 2)$ and $\alpha_{2 j}(j \geq 3)$. If $\chi\left(\alpha_{1 j}\right) \neq 0$ or $\chi\left(\alpha_{2 j}\right) \neq 0$ for some $j \geq 3$, then $\alpha_{1 j} \alpha_{2 j}$ is hyperbolic with axis $A$, so 
we arrive at a contradiction: $C_{\alpha_{12}} \supseteq A$. Hence, $\chi\left(\alpha_{1 j}\right)=0$ and $\chi\left(\alpha_{2 j}\right)=0$, for all $j \geq 3$. (1).

We have shown that $\chi\left(\alpha_{i j}\right)=0$ if $i \geq 3$ or $j \geq 3$, so our character $\chi$ is of type

Case 2. There exists a column of $M$ containing two hyperbolic generators.

Permuting the indices if necessary, we may assume that $\chi\left(\alpha_{21}\right) \neq 0$ and $\chi\left(\alpha_{31}\right) \neq$ 0 . Let $A$ be the common axis of $\alpha_{21}$ and $\alpha_{31}$. By Lemma 1. $C_{\alpha_{i j}} \supseteq A$ if $i \geq 4$ or $j \geq 4$. The only remaining generators are $\alpha_{12}, \alpha_{32}, \alpha_{13}$, and $\alpha_{23}$.

The products $\alpha_{12} \alpha_{32}$ and $\alpha_{13} \alpha_{23}$ commute with $\alpha_{31}$ and $\alpha_{21}$, respectively. Hence $C_{\alpha_{12} \alpha_{32}} \supseteq A$ and $C_{\alpha_{13} \alpha_{23}} \supseteq A$, resulting in two equivalences from Fact B:

$$
\begin{aligned}
& C_{\alpha_{12}} \supseteq A \Longleftrightarrow C_{\alpha_{32}} \supseteq A, \\
& C_{\alpha_{13}} \supseteq A \Longleftrightarrow C_{\alpha_{23}} \supseteq A .
\end{aligned}
$$

Further, each of the products $\alpha_{12} \alpha_{32}$ and $\alpha_{13} \alpha_{23}$ commutes with three of the four generators $\alpha_{12}, \alpha_{32}, \alpha_{13}, \alpha_{23}$. Therefore if either of the products is hyperbolic, then all four characteristic subtrees must contain $A$, so $A$ is invariant under the group action. This is impossible because the action is exceptional, so both of the products must be elliptic. Thus, $\chi\left(\alpha_{12}\right)=-\chi\left(\alpha_{32}\right)$ and $\chi\left(\alpha_{13}\right)=-\chi\left(\alpha_{23}\right)$.

By Lemma $3 \chi\left(\alpha_{i j}\right)=0$ if $i, j \in\{\hat{1}, \hat{2}, 3, \ldots, n\}$ or if $i, j \in\{\hat{1}, 2, \hat{3}, 4, \ldots, n\}$. By Lemma 2 and its corollary, $\chi\left(\alpha_{21}\right)=-\chi\left(\alpha_{31}\right)$ and $\chi\left(\alpha_{i 1}\right)=0$ for all $i \geq 4$.

If $\chi\left(\alpha_{1 j}\right) \neq 0$, for some $j \geq 4$, then both $\alpha_{1 j} \alpha_{2 j}$ and $\alpha_{1 j} \alpha_{3 j}$ are hyperbolic. In this case both have axis $A$, so $C_{\alpha_{12}}$ and $C_{\alpha_{13}}$ contain $A$, as well. Hence, $A$ is contained in all of the generators' characteristic subtrees, which contradicts the action's exceptionality. For all $j \geq 4$, then, $\chi\left(\alpha_{1 j}\right)=0$.

Now we have shown that $\chi\left(\alpha_{i j}\right)=0$ if $i \geq 4$ or $j \geq 4$, and that the following equations hold:

$$
\begin{aligned}
& \chi\left(\alpha_{21}\right)=-\chi\left(\alpha_{31}\right), \\
& \chi\left(\alpha_{12}\right)=-\chi\left(\alpha_{32}\right), \\
& \chi\left(\alpha_{13}\right)=-\chi\left(\alpha_{23}\right) .
\end{aligned}
$$

Thus, $\chi$ is of type (2). (2).

From these cases we conclude that every character in $\Sigma\left(P \Sigma_{n}\right)^{c}$ is of type (1) or

Proof ( $\supseteq$ ). Let $\chi: P \Sigma_{n} \rightarrow \mathbf{R}$ be a character of type (1) or (2). We must show that $\chi \notin \Sigma\left(P \Sigma_{n}\right)$. If $\chi$ factors through a free group, then it must correspond to an exceptional action on an $\mathbf{R}$-tree inherited from that free group; the group has such an action because its Bieri-Neumann-Strebel invariant is empty.

Case 1. There exist $i, j$ such that $\chi$ kills all generators except possibly $\alpha_{i j}$ and $\alpha_{j i}$.

If $i, j$ are such, then consider the homomorphism $f: P \Sigma_{n} \rightarrow F(a, b)$ given by: $\alpha_{i j} \mapsto a, \alpha_{j i} \mapsto b$, and $\alpha_{k l} \mapsto 1$ if $\{k, l\} \neq\{i, j\}$. Checking McCool's presentation of $P \Sigma_{n}$, we see that $f$ is well-defined. Further, $\chi$ factors through $F(a, b)$ via $f$.

Case 2. There exist $i, j, k$ such that $\chi\left(\alpha_{j i}\right)=-\chi\left(\alpha_{k i}\right), \chi\left(\alpha_{i j}\right)=-\chi\left(\alpha_{k j}\right)$, $\chi\left(\alpha_{i k}\right)=-\chi\left(\alpha_{j k}\right)$, and $\chi$ kills all other generators. 
If $i, j, k$ are such, then consider the homomorphism $f: P \Sigma_{n} \rightarrow F(a, b, c)$ given by:

$$
\begin{array}{ll}
\alpha_{j i} \mapsto a & \alpha_{k i} \mapsto \bar{a} \\
\alpha_{i j} \mapsto b & \alpha_{k j} \mapsto \bar{b} \\
\alpha_{i k} \mapsto c & \alpha_{j k} \mapsto \bar{c}
\end{array}
$$

with all other generators sent to 1 . Again we determine that $f$ is well-defined and that $\chi$ factors through $F(a, b, c)$ via $f$.

In either case, $\chi$ corresponds to an exceptional abelian action of $P \Sigma_{n}$ on an $\mathbf{R}$-tree. Hence, $\chi \notin \Sigma\left(P \Sigma_{n}\right)$.

\section{ACKNOWLEDGMENT}

It is a pleasure to express my gratitude to John Meier for suggesting this problem and encouraging me.

\section{REFERENCES}

[1] R. Bieri, W. D. Neumann, and R. Strebel, "A geometric invariant of discrete groups," Invent. Math. 90 (1987), 451-477. MR 89b:20108

[2] K. S. Brown, "Trees, valuations, and the Bieri-Neumann-Strebel invariant," Invent. Math. 90 (1987), 479-504. MR 89e:20060

[3] D. J. Collins, "Cohomological dimension and symmetric automorphisms of a free group," Comment. Math. Helv. 64 (1989), 44-61. MR 90e:20035

[4] M. Culler and J. Morgan, "Group actions on R-trees," Proc. London Math. Soc. 55 (1987), 571-604. MR 88f:20055

[5] D. L. Goldsmith, "The theory of motion groups," Mich. Math. J. 28 (1981), 3-17. MR 82h:57007

[6] M. Gutiérrez and S. Krstić, "Normal forms for basis-conjugating automorphisms of a free group," preprint.

[7] G. Levitt, "R-trees and the Bieri-Neumann-Strebel Invariant," Publ. Mat. 38 (1994), 195202. MR 95f:20045

[8] J. McCool, "On basis-conjugating automorphisms of free groups," Can. J. Math. 38 (1986), 1525-1529. MR 87m:20093

[9] H. Meinert, "The Bieri-Neumann-Strebel invariants for graph products of groups," J. Pure Appl. Alg. 103 (1995), 205-210. MR 96i:20047

[10] J. W. Morgan, " $\Lambda$-trees and their applications," Bull. Amer. Math. Soc. 26 (1992), 87-112. MR 92e:20017

Department of Mathematics, Cornell University, White Hall, Ithaca, New York 14853

Current address: Department of Mathematics and Statistics, University of Nebraska, Lincoln, Nebraska 68588-0323

E-mail address: lorlandi@math.unl.edu 